Optimizing stellarators for large flows

This content has been downloaded from IOPscience. Please scroll down to see the full text. 2014 Plasma Phys. Control. Fusion 56094003

(http://iopscience.iop.org/0741-3335/56/9/094003)

View the table of contents for this issue, or go to the journal homepage for more

Download details:

IP Address: 130.206.40.30

This content was downloaded on 13/08/2014 at $14: 47$

Please note that terms and conditions apply. 


\title{
Optimizing stellarators for large flows
}

\author{
Iván Calvo ${ }^{1,4}$, Felix I Parra ${ }^{2,3}$, J Arturo Alonso $^{1}$ and José Luis Velasco ${ }^{1}$ \\ ${ }^{1}$ Laboratorio Nacional de Fusión, CIEMAT, 28040 Madrid, Spain \\ ${ }^{2}$ Rudolf Peierls Centre for Theoretical Physics, University of Oxford, Oxford OX1 3NP, UK \\ ${ }^{3}$ Culham Centre for Fusion Energy, Abingdon OX14 3DB, UK \\ E-mail: ivan.calvo@ciemat.es, felix.parradiaz@physics.ox.ac.uk, arturo.alonso@externos.ciemat.es \\ and joseluis.velasco@ciemat.es
}

Received 31 January 2014, revised 4 April 2014

Accepted for publication 14 April 2014

Published 13 August 2014

\begin{abstract}
Plasma flow is damped in stellarators because they are not intrinsically ambipolar, unlike tokamaks, in which the flux-surface averaged radial electric current vanishes for any value of the radial electric field. Only quasisymmetric stellarators are intrinsically ambipolar, but exact quasisymmetry is impossible to achieve in non-axisymmetric toroidal configurations. By calculating the violation of intrinsic ambipolarity due to deviations from quasisymmetry, one can derive criteria to assess when a stellarator can be considered quasisymmetric in practice, i.e. when the flow damping is weak enough. Let us denote by $\alpha$ a small parameter that controls the size of a perturbation to an exactly quasisymmetric magnetic field. Recently, it has been shown that if the gradient of the perturbation is sufficiently small, the flux-surface averaged radial electric current scales as $\alpha^{2}$ for any value of the collisionality. It was also argued that when the gradient of the perturbation is large, the quadratic scaling is replaced by a more unfavorable one. In this paper, perturbations with large gradients are rigorously treated. In particular, it is proven that for low collisionality a perturbation with large gradient yields, at best, an $O(|\alpha|)$ deviation from quasisymmetry. Heuristic estimations in the literature incorrectly predicted an $O\left(|\alpha|^{3 / 2}\right)$ deviation.
\end{abstract}

Keywords: magnetic confinement fusion, plasma rotation, stellarator, quasisymmetry

(Some figures may appear in colour only in the online journal)

\section{Introduction}

Quasisymmetry [1,2] is an attractive property in stellarator design. It defines stellarator magnetic field configurations that make these devices behave like tokamaks to lowest order. In particular, the plasma flow is not damped in quasisymmetric stellarators. Therefore, a perfectly quasisymmetric stellarator would bring together the advantages of both concepts [3]. On the one hand, the good confinement properties and rotation capabilities of the tokamak. On the other hand, the steady state character and absence of disruptions of the stellarator. Some of the benefits of rotation, such as the stabilization of macroscopic magnetohydrodynamic modes [4], might not be as relevant in stellarators as they are in tokamaks because those modes are less worrisome in the former. But differential rotation, i.e. flow shear, can also reduce turbulent transport [5] and

\footnotetext{
${ }^{4}$ Author to whom any correspondence should be addressed.
}

this is especially important at the edge of stellarators, where transport is dominated by turbulence [6] and other stabilizing mechanisms like sheared rotational transform are typically not present. Looking for magnetic configurations that admit large flows gives a sensible path to eventually achieving large flow shear. Hence, requiring that the stellarator be quasisymmetric at least in a neighborhood of the edge seems a justified design goal.

Actually, exact quasisymmetry can only be achieved on a flux surface. Garren and Boozer proved in [7] that the rest of the plasma volume will necessarily break the quasisymmetry condition. This does not invalidate the attractiveness of quasisymmetry as a stellarator optimization concept, however, because it is possible to reach configurations reasonably close to quasisymmetric [8]. In other words, when quasisymmetry is involved, one is necessarily dealing with a magnetic field that at most has the form $\boldsymbol{B}=\boldsymbol{B}_{0}+\alpha \boldsymbol{B}_{1}$, where $\boldsymbol{B}_{0}$ is quasisymmetric and $\alpha \boldsymbol{B}_{1}$ is a small deviation from quasisymmetry. It 
seems important to understand how the desirable properties of quasisymmetric stellarators are affected by the perturbation $\alpha \boldsymbol{B}_{1}$. The appropriate analysis of this problem leads to the derivation of criteria to assess when a stellarator can be called quasisymmetric in practice. In [9] we gave one such criterion for a wide class of perturbations $\alpha \boldsymbol{B}_{1}$.

A magnetic field is quasisymmetric if and only if the fluxsurface averaged lowest-order radial electric current identically vanishes for any value of the radial electric field, and for any density and temperature profiles $[9,10]$. This feature is called intrinsic ambipolarity and can be employed as the defining property of quasisymmetry. In [9] we studied how the flux-surface averaged radial electric current goes to zero as a function of $\alpha$. We showed that, whenever the gradient of $B_{0}$ is much larger than the gradient of $\alpha B_{1}$, the flux-surface averaged radial electric current scales with $\alpha^{2}$, i.e.

$$
\langle\boldsymbol{J} \cdot \nabla \psi\rangle_{\psi} \sim \alpha^{2} k
$$

where $\boldsymbol{J}$ is the electric current density, $\psi$ is the flux-surface label coordinate, $\langle\cdot\rangle_{\psi}$ denotes the flux-surface average operation defined in section 2 and the form of the factor $k$ depends on the collisionality regime. For example,

$$
k \sim \epsilon_{\mathrm{i}}^{2} e n_{\mathrm{i}} v_{\mathrm{ti}}|\nabla \psi|
$$

when $v_{\mathrm{i}} L_{0} / v_{\mathrm{ti}} \sim 1$, whereas

$$
k \sim \frac{\epsilon_{\mathrm{i}}^{2} v_{\mathrm{ti}}}{v_{\mathrm{i}} L_{0}} e n_{\mathrm{i}} v_{\mathrm{ti}}|\nabla \psi|
$$

when $v_{\mathrm{i}} L_{0} / v_{\mathrm{ti}} \ll 1$. Here, $\epsilon_{\mathrm{i}}:=\rho_{\mathrm{i}} / L_{0}$ is the ion Larmor radius $\rho_{\mathrm{i}}$ over the typical variation length of $B_{0}, L_{0}:=\left|\nabla \ln B_{0}\right|^{-1}$, $e$ is the proton charge, $n_{\mathrm{i}}$ is the equilibrium ion density, $v_{\mathrm{ti}}$ is the ion thermal speed and $v_{\mathrm{i}}$ is the ion-ion collision frequency. It is worth being more precise about the conditions under which this quadratic scaling in $\alpha$ is obtained. Assume that $\{\psi, \Theta, \zeta\}$ are Boozer coordinates [11], which exist as long as $(\nabla \times \boldsymbol{B}) \cdot \nabla \psi=0$. It is known [10] that in these coordinates $B_{0} \equiv B_{0}(\psi, M \Theta-N \zeta)$ depends only on a single helicity $M \Theta-N \zeta$ for some pair $(M, N)$. Without loss of generality we can take $B_{1}(\psi, \Theta, \zeta)$ such that it has vanishing flux-surface average and such that it does not contain the helicity $(M, N)$. Then, the scaling equation (1) holds if

$$
\begin{aligned}
& \frac{\left|\alpha \partial_{\Theta} B_{1}\right|}{\left|\partial_{\Theta} B_{0}\right|} \sim \alpha, \\
& \frac{\left|\alpha \partial_{\zeta} B_{1}\right|}{\left|\partial_{\zeta} B_{0}\right|} \sim \alpha .
\end{aligned}
$$

References [10] and [12] proved that flows are undamped in a stellarator only if it is intrinsically ambipolar, which is equivalent to being quasisymmetric. Then, it is easy to derive a criterion for rotation from (1). This has been done in detail in [9]. The result is that when (4) holds, rotation in the symmetry direction can be close to sonic as long as

$$
|\alpha|<\epsilon_{\mathrm{i}}^{1 / 2}
$$

In order to avoid confusion, we need to discuss the result in [13]. In that reference, it has been proven that strictly sonic equilibrium flows cannot take place in a stellarator, even if it is quasisymmetric. However, the obstructions are absent if $\epsilon_{\mathrm{i}} v_{\mathrm{ti}} \ll M v_{\mathrm{ti}} \ll v_{\mathrm{ti}}$, where $M=V_{\mathrm{i}} / v_{\mathrm{ti}}$ is the Mach number and $V_{\mathrm{i}}$ is the equilibrium plasma flow velocity. This is the ordering in which our work should be understood.

It has also been argued in [9] that if (4) is not satisfied, then the scaling is expected to be more unfavorable than (1). Actually, an arbitrary perturbation that satisfies $|\alpha| B_{1} \ll B_{0}$ and, in addition, $\left|\alpha \partial_{\Theta} B_{1}\right| \sim\left|\partial_{\Theta} B_{0}\right|$ and $\left|\alpha \partial_{\zeta} B_{1}\right| \sim\left|\partial_{\zeta} B_{0}\right|$ (compare with (4)) gives a flux-surface averaged radial electric current $O\left(\alpha^{0}\right)$, and therefore the perturbed configuration is not close to quasisymmetry anymore. The reason is that when $\left|\alpha \partial_{\Theta} B_{1}\right| \sim$ $\left|\partial_{\Theta} B_{0}\right|$ or $\left|\alpha \partial_{\zeta} B_{1}\right| \sim\left|\partial_{\zeta} B_{0}\right|$ one cannot guarantee that the radial component of the magnetic drift, $v_{\psi, \sigma}:=\boldsymbol{v}_{M, \sigma} \cdot \nabla \psi$, can be expanded as $v_{\psi, \sigma}=v_{\psi, \sigma}^{(0)}+\alpha v_{\psi, \sigma}^{(1)}+\ldots$, where $v_{\psi, \sigma}-v_{\psi, \sigma}^{(0)}=$ $O(\alpha)$ and $v_{\psi, \sigma}^{(0)}$ corresponds to the quasisymmetric magnetic field. Since $v_{\psi, \sigma}$ enters the drift kinetic equation as a source term, $v_{\psi, \sigma}-v_{\psi, \sigma}^{(0)}$ will in general yield a perturbation $O\left(\alpha^{0}\right)$ of the distribution function and thus $\langle\boldsymbol{J} \cdot \nabla \psi\rangle_{\psi}=O\left(\alpha^{0}\right)$.

The above results tell us that, when designing a stellarator that intends to be quasisymmetric, it would be desirable to satisfy (1). If this is not possible due to other design constraints, the breakdown of the $\alpha^{2}$ scaling does not necessarily imply an $\alpha^{0}$ scaling. An intermediate result between the $\alpha^{2}$ and $\alpha^{0}$ scalings is obtained when $\left|\alpha \hat{\boldsymbol{b}} \cdot \nabla B_{1}\right| \sim\left|\hat{\boldsymbol{b}} \cdot \nabla B_{0}\right|$ but

$$
v_{\psi, \sigma}-v_{\psi, \sigma}^{(0)}=O(\alpha)
$$

Since $v_{\psi, \sigma}-v_{\psi, \sigma}^{(0)} \propto\left(\hat{\boldsymbol{b}} \times \nabla B_{1}\right) \cdot \nabla \psi$, condition (6) is achieved for perturbations that satisfy $\left(\hat{b} \times \nabla B_{1}\right) \cdot \nabla \psi=O(\alpha)$, i.e. the component of $\nabla B_{1}$ along the flux surface is mostly parallel to the magnetic field lines. From now on and throughout the paper, we assume that the stellarator has been designed so that (6) is satisfied. Our objective is to find out what scaling replaces (1). Advancing the final result, we will learn that for low collisionality the quadratic scaling is replaced by

$$
\langle\boldsymbol{J} \cdot \nabla \psi\rangle_{\psi} \sim \frac{|\alpha| \epsilon_{\mathrm{i}}^{2} v_{\mathrm{ti}}}{L_{0} v_{\mathrm{i}}} e n_{\mathrm{i}} v_{\mathrm{ti}}|\nabla \psi| .
$$

Equation (7) might seem surprising if one notes that $\left|\alpha \hat{\boldsymbol{b}}_{0} \cdot \nabla B_{1}\right| \sim\left|\hat{\boldsymbol{b}}_{0} \cdot \nabla B_{0}\right|$ implies that secondary wells can be created. It has typically been argued in the literature [14] (see also [15] and references therein) that these wells give a scaling of the radial fluxes with $|\alpha|^{3 / 2}$ and that they dominate transport. We will show that this is incorrect: particles trapped in secondary wells and in large wells are both associated to a $|\alpha|$ scaling.

The rest of the paper is organized as follows. Section 2 is a brief reminder of the derivation of the $\alpha^{2}$ scaling given in [9]. In section 3 we explain in more detail why this scaling can be broken when $\left|\alpha \hat{\boldsymbol{b}}_{0} \cdot \nabla B_{1}\right| \sim\left|\hat{\boldsymbol{b}}_{0} \cdot \nabla B_{0}\right|$. We also show that in order to find the scaling that replaces (1), passing particles are irrelevant and we can focus on trapped particles, distinguishing between the ones trapped in large wells and those trapped in small secondary wells. As a preliminary step, we work out the scaling of the orbit-averaged radial magnetic drift for both types of trajectories in section 4 . In section 5 we obtain the 
scaling of the distribution function and finally prove (7). We will also comment on the modification of the rotation criterion (5). The conclusions are presented in section 6 .

\section{Small helicity perturbations and $\alpha^{2}$ scaling}

In this section we present the equations involved in our problem and recall the results of section 7 in [9].

We employ phase-space coordinates $\{\boldsymbol{R}, u, \mu\}$, with $\boldsymbol{R}$ the guiding-center position, $u$ the parallel velocity and $\mu$ the magnetic moment. The drift-kinetic equations rely on the smallness of the normalized ion Larmor radius, $\epsilon_{\mathrm{i}} \ll 1$. The distribution function is expanded as $F_{\sigma}=F_{\sigma 0}+F_{\sigma 1}+$ $O\left(\epsilon_{\sigma}^{2} F_{\sigma 0}\right)$, with $F_{\sigma 1} / F_{\sigma 0}=O\left(\epsilon_{\sigma}\right)$. Here, $\epsilon_{\sigma}=\rho_{\sigma} / L_{0}$ is the ratio of the Larmor radius of species $\sigma$ over the typical variation length of $B_{0}$. The condition $\epsilon_{\sigma} \ll 1$ means that species $\sigma$ is strongly magnetized. The electrostatic potential is expressed as $\varphi=\varphi_{0}+\varphi_{1}+O\left(\epsilon_{\mathrm{i}}^{2} T_{\mathrm{i}} / e\right)$, where $\varphi_{1} / \varphi_{0}=O\left(\epsilon_{\mathrm{i}}\right)$. We adopt a maximal expansion in which $v_{* \sigma} \sim 1$, where $v_{* \sigma}=v_{\sigma} L_{0} / v_{t \sigma}$ is the collisionality of species $\sigma, v_{t \sigma}$ is the thermal speed, and $v_{\sigma}=\sum_{\sigma^{\prime}} v_{\sigma \sigma^{\prime}}, v_{\sigma \sigma^{\prime}}$ is the frequency of collisions between species $\sigma$ and $\sigma^{\prime}$. To lowest order in $\epsilon_{\mathrm{i}}$ we deduce that $\varphi_{0}$ only depends on $\psi$ and that $F_{\sigma 0}$ is Maxwellian,

$F_{\sigma 0}(\boldsymbol{R}, u, \mu)=n_{\sigma}\left(\frac{m_{\sigma}}{2 \pi T_{\sigma}}\right)^{3 / 2} \exp \left(-\frac{m_{\sigma}\left(u^{2} / 2+\mu B\right)}{T_{\sigma}}\right)$,

where $m_{\sigma}$ is the mass of species $\sigma$, the density $n_{\sigma}$ and temperature $T_{\sigma}$ depend only on $\psi$, and $T_{\sigma}=T_{\sigma^{\prime}}$ for every pair $\sigma, \sigma^{\prime}$ (the ion and electron temperatures can be decoupled if a mass ratio expansion $\sqrt{m_{\mathrm{e}} / m_{\mathrm{i}}} \ll 1$ is performed). The densities satisfy the lowest-order quasineutrality equation, $\sum_{\sigma} Z_{\sigma} e n_{\sigma}=0$.

Define the non-adiabatic piece of the distribution function by $G_{\sigma 1}:=F_{\sigma 1}+\left(Z_{\sigma} e \varphi_{1} / T_{\sigma}\right) F_{\sigma 0}$. It satisfies the drift kinetic equation

$$
\begin{aligned}
& \left(u \hat{\boldsymbol{b}} \cdot \nabla-\mu \hat{\boldsymbol{b}} \cdot \nabla B \partial_{u}\right) G_{\sigma 1} \\
& \quad+\Upsilon_{\sigma} \boldsymbol{v}_{M, \sigma} \cdot \nabla \psi F_{\sigma 0}=C_{\sigma}^{\ell}\left[G_{1}\right] .
\end{aligned}
$$

Here, $C_{\sigma}^{\ell}\left[G_{1}\right]$ is the linearized Fokker-Planck collision operator,

$$
\boldsymbol{v}_{M, \sigma}=\frac{1}{\Omega_{\sigma}} \hat{\boldsymbol{b}} \times\left(u^{2} \kappa+\mu \nabla B\right)
$$

is the magnetic-drift velocity, $\Omega_{\sigma}=Z_{\sigma} e B /\left(m_{\sigma} c\right)$ is the gyrofrequency of species $\sigma, c$ is the speed of light, and

$$
\begin{aligned}
\Upsilon_{\sigma} & :=\frac{Z_{\sigma} e}{T_{\sigma}} \partial_{\psi} \varphi_{0}+\frac{1}{n_{\sigma}} \partial_{\psi} n_{\sigma} \\
& +\left(\frac{m_{\sigma}\left(u^{2} / 2+\mu B\right)}{T_{\sigma}}-\frac{3}{2}\right) \frac{1}{T_{\sigma}} \partial_{\psi} T_{\sigma} .
\end{aligned}
$$

The neoclassical expression for the flux-surface average of the radial electric current reads

$$
\langle\boldsymbol{J} \cdot \nabla \psi\rangle_{\psi}=2 \pi\left\langle\sum_{\sigma} Z_{\sigma} e \int B \boldsymbol{v}_{M, \sigma} \cdot \nabla \psi G_{\sigma 1} \mathrm{~d} u \mathrm{~d} \mu\right\rangle_{\psi},
$$

where $Z_{\sigma} e$ is the charge of species $\sigma$. The flux-surface average of a function $f(\psi, \Theta, \zeta)$ is

$$
\langle f\rangle_{\psi}=\frac{1}{V^{\prime}} \int_{0}^{2 \pi} \int_{0}^{2 \pi} \sqrt{g} f \mathrm{~d} \Theta \mathrm{d} \zeta
$$

For the moment, $\{\psi, \Theta, \zeta\}$ are arbitrary flux coordinates, $\sqrt{g}$ is the square root of the metric determinant, $V(\psi)$ is the plasma volume enclosed by the surface labeled by $\psi$ and its derivative is given by

$$
V^{\prime}(\psi)=\int_{0}^{2 \pi} \int_{0}^{2 \pi} \sqrt{g} \mathrm{~d} \Theta \mathrm{d} \zeta .
$$

The ambipolarity condition,

$$
2 \pi\left\langle\sum_{\sigma} Z_{\sigma} e \int B \boldsymbol{v}_{M, \sigma} \cdot \nabla \psi G_{\sigma 1} \mathrm{~d} u \mathrm{~d} \mu\right\rangle_{\psi}=0,
$$

imposes $\langle\boldsymbol{J} \cdot \nabla \psi\rangle_{\psi}$ to vanish to lowest order in $\epsilon_{\mathrm{i}}$. Equations (9) and (15) are the relevant ones in stellarator neoclassical calculations ${ }^{5}$.

We write our magnetic field as $\boldsymbol{B}=\boldsymbol{B}_{0}+\alpha \boldsymbol{B}_{1}$, where $\boldsymbol{B}_{0}$ is quasisymmetric and $\alpha \boldsymbol{B}_{1}$ is a small perturbation. We assume that $\{\psi, \Theta, \zeta\}$ are Boozer coordinates and take $B_{1}$ as explained below equation (3). We want to show that if (4) is satisfied, then $\langle\boldsymbol{J} \cdot \nabla \psi\rangle_{\psi}=O\left(\alpha^{2}\right)$. We recall that in Boozer coordinates $\boldsymbol{B}$ can be written as

$$
\boldsymbol{B}=-\tilde{\eta} \nabla \psi+\frac{I(\psi)}{2 \pi} \nabla \Theta+\frac{J(\psi)}{2 \pi} \nabla \zeta
$$

and as

$$
\boldsymbol{B}=\frac{\Psi_{p}^{\prime}(\psi)}{2 \pi} \nabla \zeta \times \nabla \psi+\frac{\Psi_{t}^{\prime}(\psi)}{2 \pi} \nabla \psi \times \nabla \Theta .
$$

The prime denotes differentiation with respect to $\psi, \Psi_{t}$ is the toroidal flux, $\Psi_{p}$ the poloidal flux, and $\tilde{\eta}(\psi, \Theta, \zeta)$ is a singlyvalued function. An important property of Boozer coordinates is that $\sqrt{g}$ can be expressed in terms of the magnitude of the magnetic field,

$$
\sqrt{g}=\frac{V^{\prime}\left\langle B^{2}\right\rangle_{\psi}}{4 \pi^{2} B^{2}}
$$

The following related identity will be useful later on. Namely,

$$
\hat{\boldsymbol{b}} \cdot \nabla \Theta=\frac{2 \pi \Psi_{p}^{\prime} B}{V^{\prime}\left\langle B^{2}\right\rangle_{\psi}} .
$$

The derivative along the magnetic field reads

$$
\hat{\boldsymbol{b}} \cdot \nabla=\frac{2 \pi \Psi_{t}^{\prime} B}{\left\langle B^{2}\right\rangle_{\psi} V^{\prime}}\left(t \partial_{\Theta}+\partial_{\zeta}\right)
$$

where $t(\psi)=\Psi_{p}^{\prime}(\psi) / \Psi_{t}^{\prime}(\psi)$ is the rotational transform. Finally, the radial component of the magnetic drift is given by $v_{\psi, \sigma}:=v_{M, \sigma} \cdot \nabla \psi=\frac{2 \pi m_{\sigma} c\left(u^{2}+\mu B\right)}{Z_{\sigma} e V^{\prime}\left\langle B^{2}\right\rangle_{\psi} B}\left(I \partial_{\zeta} B-J \partial_{\Theta} B\right)$,

5 To be precise, the neoclassical description of some low collisionality stellarator regimes, such as the $\sqrt{v}$ and $v$ regimes, requires additional terms in (9). The reason is that at low collisionality $G_{\sigma 1}$ scales with $v_{* \sigma}^{-1}$ and terms that are nominally of higher order in the $\epsilon_{\sigma}$ expansion of the drift-kinetic equation may actually be non-negligible. 
where $(\nabla \times \boldsymbol{B}) \cdot \nabla \psi \equiv 0$ has been used. Therefore,

$$
\begin{aligned}
\langle\boldsymbol{J} \cdot & \nabla \psi\rangle_{\psi}=\sum_{\sigma} \frac{m_{\sigma} c}{V^{\prime}} \int_{0}^{2 \pi} \int_{0}^{2 \pi} \mathrm{d} \Theta \mathrm{d} \zeta \\
& \times \int \frac{u^{2}+\mu B}{B^{2}}\left(I \partial_{\zeta} B-J \partial_{\Theta} B\right) G_{\sigma 1} \mathrm{~d} u \mathrm{~d} \mu .
\end{aligned}
$$

Observe equations (9), (20), (21), (22), and recall that the kernel of the collision operator in drift-kinetic coordinates depends on the magnetic field exclusively through $B$ (see appendix $\mathrm{G}$ of [9]). Then, the magnetic geometry information enters the drift kinetic equation and the ambipolarity condition only via the function $B(\psi, \Theta, \zeta)$.

As stated in the Introduction, in Boozer coordinates $B_{0}$ depends only on a single helicity $M \Theta-N \zeta$. It is enough to carry out the proof for quasi-axisymmetric $B_{0}$, i.e. $\partial_{\zeta} B_{0} \equiv 0$, which corresponds to $N=0$. If $\boldsymbol{B}_{0}$ is helically symmetric, $N \neq 0$, the problem may be reduced to the quasi-axisymmetric case by a change of Boozer angles. Specifically, one can define $\bar{\Theta}:=M \Theta-N \zeta$ and employ $\{\psi, \bar{\Theta}, \zeta\}$ as Boozer coordinates.

Since $B_{1}$ can be chosen such that $\left\langle B_{1}\right\rangle_{\psi}=0$ and such that it does not contain the helicity of $B_{0}$, we have, in this case,

$$
\int_{0}^{2 \pi} B_{1}(\psi, \Theta, \zeta) \mathrm{d} \zeta=0 .
$$

Now, we are ready to calculate the scaling of (22). Since (4) holds, every term on the right side of (22) can be expanded in integer powers of $\alpha$. The $O\left(\alpha^{0}\right)$ terms vanish due to quasisymmetry. The $O(\alpha)$ terms, $\langle\boldsymbol{J} \cdot \nabla \psi\rangle_{\psi}^{(1)}$, are

$$
\begin{aligned}
& \langle\boldsymbol{J} \cdot \nabla \psi\rangle_{\psi}^{(1)} \\
& =-\sum_{\sigma} \frac{m_{\sigma} c}{V^{\prime}} \int_{0}^{2 \pi} \int_{0}^{2 \pi} \mathrm{d} \Theta \mathrm{d} \zeta \int \frac{u^{2}+\mu B_{0}}{B_{0}^{2}} J \partial_{\Theta} B_{0} G_{\sigma}^{(1)} \mathrm{d} u \mathrm{~d} \mu \\
& +\sum_{\sigma} \frac{m_{\sigma} c}{V^{\prime}} \int_{0}^{2 \pi} \int_{0}^{2 \pi} \mathrm{d} \Theta \mathrm{d} \zeta \int\left[\frac{2 u^{2}+\mu B_{0}}{B_{0}^{3}} B_{1} J \partial_{\Theta} B_{0}\right. \\
& \left.+\frac{u^{2}+\mu B_{0}}{B_{0}^{2}}\left(I \partial_{\zeta} B_{1}-J \partial_{\Theta} B_{1}\right)\right] G_{\sigma}^{(0)} \mathrm{d} u \mathrm{~d} \mu,
\end{aligned}
$$

where

$$
G_{\sigma 1}:=G_{\sigma}^{(0)}+\alpha G_{\sigma}^{(1)}+O\left(\alpha^{2}\right)
$$

The equations determining $G_{\sigma}^{(0)}$ and $G_{\sigma}^{(1)}$ are

$$
\begin{aligned}
(u \hat{\boldsymbol{b}} & \left.\cdot \nabla-\mu \hat{\boldsymbol{b}} \cdot \nabla B \partial_{u}\right)^{(0)} G_{\sigma}^{(0)}+\left(\Upsilon_{\sigma} v_{\psi, \sigma} F_{\sigma 0}\right)^{(0)} \\
& =C_{\sigma}^{\ell(0)}\left[G^{(0)}\right]
\end{aligned}
$$

and

$$
\begin{aligned}
(u \hat{\boldsymbol{b}} & \left.\cdot \nabla-\mu \hat{\boldsymbol{b}} \cdot \nabla B \partial_{u}\right)^{(0)} G_{\sigma}^{(1)} \\
& +\left(u \hat{\boldsymbol{b}} \cdot \nabla-\mu \hat{\boldsymbol{b}} \cdot \nabla B \partial_{u}\right)^{(1)} G_{\sigma}^{(0)} \\
& +\left(\Upsilon_{\sigma} v_{\psi, \sigma} F_{\sigma 0}\right)^{(1)}=C_{\sigma}^{\ell(1)}\left[G^{(0)}\right]+C_{\sigma}^{\ell(0)}\left[G^{(1)}\right] .
\end{aligned}
$$

We have employed the notation

$$
(\hat{\boldsymbol{b}} \cdot \nabla)^{(0)}=\frac{2 \pi \Psi_{t}^{\prime} B_{0}}{\left\langle B^{2}\right\rangle_{\psi} V^{\prime}}\left(t \partial_{\Theta}+\partial_{\zeta}\right),
$$

$(\hat{\boldsymbol{b}} \cdot \nabla)^{(1)}=\frac{2 \pi \Psi_{t}^{\prime} B_{1}}{\left\langle B^{2}\right\rangle_{\psi} V^{\prime}}\left(t \partial_{\Theta}+\partial_{\zeta}\right)$,

$(\hat{\boldsymbol{b}} \cdot \nabla B)^{(0)}=\frac{2 \pi \Psi_{t}^{\prime} B_{0}}{\left\langle B^{2}\right\rangle_{\psi} V^{\prime}} t \partial_{\Theta} B_{0}$,

$(\hat{\boldsymbol{b}} \cdot \nabla B)^{(1)}=\frac{2 \pi \Psi_{t}^{\prime}}{\left\langle B^{2}\right\rangle_{\psi} V^{\prime}}\left[B_{1} t \partial_{\Theta} B_{0}+B_{0}\left(t \partial_{\Theta}+\partial_{\zeta}\right) B_{1}\right]$,

etc. $C_{\sigma}^{\ell(0)}$ is the linearized collision operator corresponding to $B_{0}$ and $C_{\sigma}^{\ell(1)}$ is the first-order correction. Their explicit expressions are not needed here.

From (26) and (27), one obtains

$$
\partial_{\zeta} G_{\sigma}^{(0)}=0
$$

and

$$
\int_{0}^{2 \pi} G_{\sigma}^{(1)} \mathrm{d} \zeta=0
$$

Therefore, each term on the right-hand side of (24) can be written as

$$
\int_{0}^{2 \pi} \int_{0}^{2 \pi} q(\psi, \Theta) f(\psi, \Theta, \zeta) \mathrm{d} \Theta \mathrm{d} \zeta
$$

for some function $f(\psi, \Theta, \zeta)$ with zero average over $\zeta$,

$$
\int_{0}^{2 \pi} f(\psi, \Theta, \zeta) \mathrm{d} \zeta=0 .
$$

Then, $\langle\boldsymbol{J} \cdot \nabla \psi\rangle_{\psi}^{(1)} \equiv 0$ follows. The quadratic terms, $\langle\boldsymbol{J} \cdot \nabla \psi\rangle_{\psi}^{(2)}$, are non-zero in general and we obtain (1). It is important to emphasize that the result is valid for any value of the collisionality. The same scaling was obtained in [16] for highly-collisional plasmas.

\section{Breakdown of the $\alpha^{2}$ scaling}

Clearly, the procedure followed in section 2 may fail if

$$
\left|\alpha \hat{\boldsymbol{b}}_{0} \cdot \nabla B_{1}\right| \sim\left|\hat{\boldsymbol{b}}_{0} \cdot \nabla B_{0}\right|
$$

because the parallel streaming operator appearing in the drift kinetic equation (9) cannot be expanded in powers of $\alpha$. Of course, (36) holds for any perturbation $\alpha \boldsymbol{B}_{1}$ near points where $\hat{\boldsymbol{b}}_{0} \cdot \nabla B_{0}=0$. The trajectories that can be affected more severely correspond to almost trapped, barely trapped, and deeply trapped particles in the magnetic field $\boldsymbol{B}_{0}$. However, it has been proven in [9] that they contribute with terms $O\left(|\alpha|^{5 / 2}\right)$ to the radial electric current, and thus are subdominant with respect to $O\left(\alpha^{2}\right)$ terms. In a sense, these trajectories, even though they satisfy (36), are trivial to treat.

Recall that $L_{0}$ is the characteristic variation length of $B_{0}$. We have to worry about stellarators where (36) happens due to $\left|\alpha \hat{\boldsymbol{b}}_{0} \cdot \nabla B_{1}\right| \sim B_{0} L_{0}^{-1}$, i.e. we are not worried about the neighborhood of a point with $\hat{b}_{0} \cdot \nabla B_{0}=0$. We can equivalently say that we have to analyze what happens when $\left|\alpha \hat{\boldsymbol{b}}_{0} \cdot \nabla B_{1}\right| \sim\left|\hat{\boldsymbol{b}}_{0} \cdot \nabla B_{0}\right|$ because the perturbation is such that

$$
\frac{L_{1}}{L_{0}} \sim|\alpha|,
$$

$L_{1}$ being the characteristic variation length of $B_{1}$. 
Collisionless particles are expected to be the most dangerous ones, and we focus on them in this paper by studying the so-called $1 / v$ regime. In the absence of collisions the kinetic energy $\varepsilon=u^{2} / 2+\mu B$ is a constant of the motion, and to lowest order in $\epsilon_{\sigma}$ particle trajectories lie on magnetic field lines. This is why it will be useful to employ the phase-space coordinates $\{\psi, \chi, \Theta, \varepsilon, \mu, s\}$, being $s=-1,1$ the sign of the parallel velocity and $\chi:=\Theta-t \zeta, \chi \in[0,2 \pi)$, a coordinate that locally labels magnetic field lines. Consider the following expansion,

$$
G_{\sigma 1}=G_{\sigma}^{[-1]}+G_{\sigma}^{[0]}+O\left(v_{* \sigma} \epsilon_{\sigma} F_{\sigma 0}\right),
$$

where $G_{\sigma}^{[j]}=O\left(v_{* \sigma}^{j} \epsilon_{\sigma} F_{\sigma 0}\right)$. Equation (9) to lowest order in $v_{* \sigma} \ll 1$ reads

$$
v_{\|} \hat{\boldsymbol{b}} \cdot \nabla \Theta \partial_{\Theta} G_{\sigma}^{[-1]}=0 .
$$

Hence, for passing particles $G_{\sigma}^{[-1]}$ is a flux function whereas for trapped particles $\overline{G_{\sigma}^{[-1]}}=G_{\sigma}^{[-1]}$; that is, for trapped particles $G_{\sigma}^{[-1]}$ is not a flux function but it is constant along the lowest order trajectories. Here,

$$
\bar{f}=\tau_{b}^{-1} \oint \frac{f(\psi, \chi, \Theta, \varepsilon, \mu, s)}{v_{\|} \hat{\boldsymbol{b}} \cdot \nabla \Theta} \mathrm{d} \Theta,
$$

with

$$
\tau_{b}=\oint \frac{1}{v_{\|} \hat{\boldsymbol{b}} \cdot \nabla \Theta} \mathrm{d} \Theta,
$$

defines the bounce average of the phase-space function $f(\psi, \chi, \Theta, \varepsilon, \mu, s)$. This is a time average over the lowest order trapped particle trajectories, that are closed, and $\tau_{b}$ is the bounce time. The angle $\Theta$ parameterizes the trajectory. In (40), the parallel velocity $v_{\|}$is to be viewed as a function of the independent variables $\{\psi, \chi, \Theta, \varepsilon, \mu, s\}$. Namely,

$$
v_{\|}(\psi, \chi, \Theta, \varepsilon, \mu, s)=s \sqrt{2(\varepsilon-\mu B(\psi, \chi, \Theta))} .
$$

We also point out that if $\{\psi, \Theta, \zeta\}$ are Boozer coordinates and $\chi=\Theta-t \zeta$, the flux surface average of a function $f(\psi, \chi, \Theta)$ reads

$$
\langle f\rangle_{\psi}=\frac{\left\langle B^{2}\right\rangle_{\psi}}{4 \pi^{2} t} \int_{0}^{2 \pi} \int_{0}^{2 \pi} \frac{1}{B^{2}} f(\psi, \chi, \Theta) \mathrm{d} \chi \mathrm{d} \Theta .
$$

To an order higher in $v_{* \sigma}$ than (39), the transit average of the Fokker-Planck equation (9) gives, for trapped particles,

$$
\Upsilon_{\sigma} \overline{v_{\psi, \sigma}} F_{\sigma 0}=\overline{C_{\sigma}^{\ell}\left[G^{[-1]}\right]} .
$$

We proceed to prove that one only has to deal with trapped trajectories because $G_{\sigma}^{[-1]}=0$ for passing particles up to terms $O\left(\alpha v_{* \sigma}^{-1} \epsilon_{\sigma} F_{\sigma 0}\right)$. Let us denote by $G_{\sigma, p}^{[-1]}$ and $G_{\sigma, t}^{[-1]}$ the distribution function $G_{\sigma}^{[-1]}$ in the passing and trapped regions, respectively. $G_{\sigma, p}^{[-1]}$ is a flux function, whereas $G_{\sigma, t}^{[-1]}$ is constant over orbits. One can write

$$
G_{\sigma}^{[-1]}(\psi, \chi, \varepsilon, \mu)=g_{\sigma}(\psi, \varepsilon, \mu)+\partial_{\chi} h_{\sigma}(\psi, \chi, \varepsilon, \mu),
$$

where $h_{\sigma}$ vanishes in the passing region, $h_{\sigma, p} \equiv 0$. Observe that by multiplying (9) by $g_{\sigma} / F_{\sigma 0}$, integrating over velocity and flux-surface averaging, we can derive the condition

$$
\left\langle\sum_{\sigma} \int \frac{g_{\sigma}}{F_{\sigma 0}} C_{\sigma}^{\ell}\left[g_{\sigma}+\partial_{\chi} h_{\sigma}\right] \mathrm{d}^{3} v\right\rangle_{\psi}=0,
$$

where we have used that for any function $Q(\psi, \varepsilon, \mu)$,

$$
\left\langle\int v_{\psi, \sigma} Q(\psi, \varepsilon, \mu) \mathrm{d}^{3} v\right\rangle_{\psi}=0
$$

The proof of (47) can be found, for example, in section 5.1 of [9]. Here, we have abbreviated $\int(\ldots) \mathrm{d}^{3} v \equiv$ $\sum_{s} \int_{0}^{\infty} \int_{0}^{\infty} 2 \pi H(\varepsilon-\mu B) B\left|v_{\|}\right|^{-1}(\ldots) \mathrm{d} \varepsilon \mathrm{d} \mu$, where $H$ is the Heaviside step function.

The kernel of the collision operator in drift-kinetic coordinates [9] depends on the spatial coordinates only via $B$, which is simply a function of $\Theta$ up to terms $O(\alpha)$ because $B_{0}$ is quasi-axisymmetric. Hence, up to $O(\alpha)$ corrections, (46) becomes

$$
\left\langle\sum_{\sigma} \int \frac{g_{\sigma}}{F_{\sigma 0}} C_{\sigma}^{\ell}\left[g_{\sigma}\right] \mathrm{d}^{3} v\right\rangle_{\psi}=0,
$$

where periodicity in $\chi$ has been employed. Equation (48) can be viewed as an entropy production condition on $g_{\sigma}$, implying that

$$
g_{\sigma}=\left(a_{\sigma, 0}(\boldsymbol{R})+m_{\sigma} a_{1}(\boldsymbol{R}) v_{\|}+m_{\sigma} a_{2}(\boldsymbol{R}) \varepsilon\right) F_{\sigma 0},
$$

where we have used that $G_{\sigma 1}$ has to be independent of the gyrophase [9]. Since $g_{\sigma}$ must be a flux function, $a_{\sigma, 0} \equiv$ $a_{\sigma, 0}(\psi), a_{2} \equiv a_{2}(\psi)$ and $a_{1} \equiv 0$.

One can always choose the flux-surface averaged densities and total energy to be given only by the $O\left(\epsilon_{\sigma}^{0}\right)$ distribution function, $F_{\sigma 0}$. Thus, we impose

$$
\left\langle\int G_{\sigma}^{[-1]} \mathrm{d}^{3} v\right\rangle_{\psi}=0 \text { for every } \sigma
$$

and

$$
\left\langle\sum_{\sigma} \int m_{\sigma} \varepsilon G_{\sigma}^{[-1]} \mathrm{d}^{3} v\right\rangle_{\psi}=0 .
$$

To lowest order in $\alpha$, this implies $a_{\sigma, 0} \equiv 0$ and $a_{2} \equiv 0$. Then, we deduce that $g_{\sigma} \equiv 0$. Since $h_{\sigma}$ vanishes in the passing region, we have obtained that $G_{\sigma, p}^{[-1]} \equiv 0$ up to $O(\alpha)$ corrections. Then, to lowest order in $\alpha$, passing particles only enter the problem by setting a vanishing boundary condition for $G_{\sigma, t}^{[-1]}$ at the interface between the passing and trapped regions.

Finding out how the solution of equation (44) depends on $\alpha$ when $\alpha B_{1}$ has a large parallel gradient will be the objective of the following sections.

\section{Scaling of the bounce-averaged radial magnetic drift in the presence of large helicity perturbations}

As a previous step to finding out how the solution of equation (44) scales with $\alpha$ when $L_{1} \sim|\alpha| L_{0}$, we investigate the scaling of $\overline{v_{\psi, \sigma}}$. At the end of section 3 we have shown that only trapped particles require detailed analysis, and consequently we focus on them. A sketch of a perturbation $\alpha B_{1}$ with large parallel gradient is shown in figure 1 . We have to distinguish 


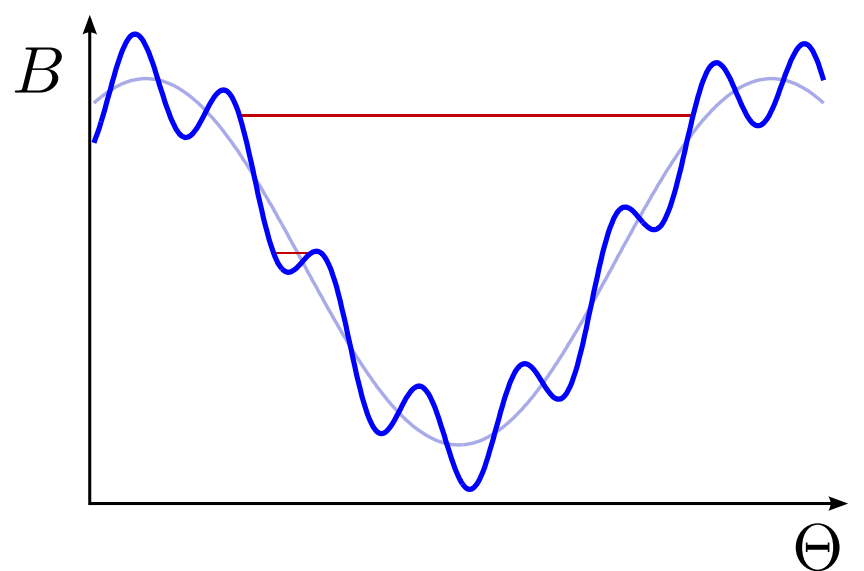

Figure 1. Dependence of the magnetic field magnitude on $\Theta$ along a magnetic field line in a quasisymmetric configuration (light) and in a quasisymmetric configuration with a large helicity perturbation added (dark). The upper red line corresponds to the trajectory of a particle trapped in a well of size $L_{0}$. The lower one corresponds to a particle trapped in a secondary well of size $L_{1}$.

two cases: a particle trapped in a well of size $L_{0}$ and a particle trapped in a secondary well of size $L_{1} \sim|\alpha| L_{0}$. Before starting the analysis of the scaling of $\overline{v_{\psi, \sigma}}$, we remind the reader that along this paper we assume that the radial magnetic drift can be expanded in integer powers of $\alpha$. Namely,

$$
v_{\psi, \sigma}-v_{\psi, \sigma}^{(0)}=O(\alpha),
$$

where $v_{\psi, \sigma}^{(0)}$ is the radial magnetic drift corresponding to $\boldsymbol{B}_{0}$. As argued in the Introduction, equation (52) should be a design criterion for quasisymmetric stellarators.

\subsection{Large wells}

Take values of energy and magnetic moment such that the particle is trapped in a well of size $L_{0}$ (see the upper red line in figure 1). In this subsection we will prove that for this particle

$$
\overline{v_{\psi, \sigma}} \sim|\alpha|^{1 / 2} \epsilon_{\sigma} v_{t \sigma}|\nabla \psi| .
$$

Because of (52), we may write

$$
\overline{v_{\psi, \sigma}}=\frac{2 \int_{\Theta_{1}}^{\Theta_{2}} v_{\psi, \sigma}^{(0)}\left[\left|v_{\|}\right|(\hat{\boldsymbol{b}} \cdot \nabla \Theta)^{(0)}\right]^{-1} \mathrm{~d} \Theta+O(\alpha)}{2 \int_{\Theta_{1}}^{\Theta_{2}}\left[\left|v_{\|}\right| \hat{\boldsymbol{b}} \cdot \nabla \Theta\right]^{-1} \mathrm{~d} \Theta} .
$$

Here, we denote by $\Theta_{1}$ and $\Theta_{2}$ the bounce points of the orbit; that is, the solutions of $\varepsilon-\mu B(\psi, \chi, \Theta)=0$. Whereas the denominator of the right side of (54) is $O\left(L_{0} / v_{t \sigma}\right)$, the integral in the numerator is dominated by a region near the endpoints whose size is $O(\alpha)$, that yields the scaling (53). Next, we proceed to give the proof.

Recalling (21), we get

$$
v_{\psi, \sigma}^{(0)}=-\frac{2 \pi m_{\sigma} c\left(2 \varepsilon-\mu B_{0}\right)}{Z_{\sigma} e V^{\prime}\left\langle B_{0}^{2}\right\rangle_{\psi} B_{0}} J \partial_{\Theta} B_{0},
$$

and using (19) we obtain

$$
(\hat{\boldsymbol{b}} \cdot \nabla \Theta)^{(0)}=\frac{2 \pi \Psi_{p}^{\prime} B_{0}}{V^{\prime}\left\langle B_{0}^{2}\right\rangle_{\psi}} .
$$

The first term in the numerator of (54) can then be recast as

$$
\begin{aligned}
2 \int_{\Theta_{1}}^{\Theta_{2}} & v_{\psi, \sigma}^{(0)}\left[\left|v_{\|}\right|(\hat{\boldsymbol{b}} \cdot \nabla \Theta)^{(0)}\right]^{-1} \mathrm{~d} \Theta \\
= & -\frac{2 m_{\sigma} c J}{Z_{\sigma} e \Psi_{p}^{\prime}} \int_{\Theta_{1}}^{\Theta_{2}} \frac{\partial_{\Theta} B_{0}(\psi, \Theta)}{B_{0}^{2}(\psi, \Theta)} \\
& \times \frac{2 \varepsilon-\mu B_{0}(\psi, \Theta)}{\sqrt{2\left(\varepsilon-\mu B_{0}(\psi, \Theta)-\mu \alpha B_{1}(\psi, \chi, \Theta)\right.}} \mathrm{d} \Theta .
\end{aligned}
$$

To simplify the notation, in what follows either we will omit the arguments of $B_{0}$ and $B_{1}$ or we will only specify the dependence on $\Theta$.

The identity

$$
-\frac{2 m_{\sigma} c J}{Z_{\sigma} e \Psi_{p}^{\prime}} \int_{\Theta_{1}}^{\Theta_{2}} \frac{\partial_{\Theta} \tilde{B}}{\tilde{B}^{2}} \frac{2 \varepsilon-\mu \tilde{B}}{\sqrt{2(\varepsilon-\mu \tilde{B})}} \mathrm{d} \Theta=0
$$

with

$$
\tilde{B}(\Theta):=B_{0}(\Theta)+\alpha B_{1}\left(\Theta_{2}\right) \frac{\Theta-\Theta_{1}}{\Theta_{2}-\Theta_{1}}+\alpha B_{1}\left(\Theta_{1}\right) \frac{\Theta_{2}-\Theta}{\Theta_{2}-\Theta_{1}}
$$

allows us to rewrite (57) as

$$
\begin{aligned}
& 2 \int_{\Theta_{1}}^{\Theta_{2}} v_{\psi, \sigma}^{(0)}\left[\left|v_{\|}\right|(\hat{\boldsymbol{b}} \cdot \nabla \Theta)^{(0)}\right]^{-1} \mathrm{~d} \Theta= \\
& \quad-2 \frac{m_{\sigma} c J}{Z_{\sigma} e \Psi_{p}^{\prime}} \int_{\Theta_{1}}^{\Theta_{2}}\left(\frac{\partial_{\Theta} B_{0}}{B_{0}^{2}} \frac{2 \varepsilon-\mu B_{0}}{\sqrt{2\left(\varepsilon-\mu B_{0}-\mu \alpha B_{1}\right)}}\right. \\
& \left.\quad-\frac{\partial_{\Theta} \tilde{B}}{\tilde{B}^{2}} \frac{2 \varepsilon-\mu \tilde{B}}{\sqrt{2(\varepsilon-\mu \tilde{B})}}\right) \mathrm{d} \Theta .
\end{aligned}
$$

We want to prove that this integral is dominated by a neighborhood of the endpoints $\Theta_{1}$ and $\Theta_{2}$ and that it scales with $|\alpha|^{1 / 2}$.

Take $K>1$ and choose $\Theta_{1}^{\prime}$ and $\Theta_{2}^{\prime},\left[\Theta_{1}^{\prime}, \Theta_{2}^{\prime}\right] \subset\left[\Theta_{1}, \Theta_{2}\right]$, such that $\varepsilon-\mu B_{0}(\Theta)>|\alpha| K$ when $\Theta \in\left[\Theta_{1}^{\prime}, \Theta_{2}^{\prime}\right]$. It is convenient to select $\Theta_{1}^{\prime}$ and $\Theta_{2}^{\prime}$ such that $\tilde{B}\left(\Theta_{1}^{\prime}\right)=\tilde{B}\left(\Theta_{2}^{\prime}\right)$. First, we show that in (60) the piece of the integral that runs over $\left[\Theta_{1}^{\prime}, \Theta_{2}^{\prime}\right]$ is negligible compared to $|\alpha|^{1 / 2}$.

Using that $\tilde{B}\left(\Theta_{1}^{\prime}\right)=\tilde{B}\left(\Theta_{2}^{\prime}\right)$, we have

$$
-\frac{2 m_{\sigma} c J}{Z_{\sigma} e \Psi_{p}^{\prime}} \int_{\Theta_{1}^{\prime}}^{\Theta_{2}^{\prime}} \frac{\partial_{\Theta} \tilde{B}}{\tilde{B}^{2}} \frac{2 \varepsilon-\mu \tilde{B}}{\sqrt{2(\varepsilon-\mu \tilde{B})}} \mathrm{d} \Theta=0 .
$$

Proving that the integration over $\left[\Theta_{1}^{\prime}, \Theta_{2}^{\prime}\right]$ in the first term of (60) is small requires some work. To fix ideas, assume that

$$
\int_{\Theta_{1}^{\prime}}^{\Theta_{2}^{\prime}} \frac{\partial_{\Theta} B_{0}}{B_{0}^{2}} \frac{2 \varepsilon-\mu B_{0}}{\sqrt{2\left(\varepsilon-\mu B_{0}-\mu \alpha B_{1}\right)}} \mathrm{d} \Theta
$$

is positive (if it is negative, the treatment is almost identical). Then,

$$
\begin{aligned}
\int_{\Theta_{1}^{\prime}}^{\Theta_{2}^{\prime}} & \frac{\partial_{\Theta} B_{0}}{B_{0}^{2}} \frac{2 \varepsilon-\mu B_{0}}{\sqrt{2\left(\varepsilon-\mu B_{0}-\mu \alpha B_{1}\right)}} \mathrm{d} \Theta \\
\leqslant & \int_{\Theta_{1}^{\prime}}^{\Theta_{m}} \frac{\partial_{\Theta} B_{0}}{B_{0}^{2}} \frac{2 \varepsilon-\mu B_{0}}{\sqrt{2\left(\varepsilon-\mu B_{0}-\mu \alpha B_{1, \min }\right)}} \mathrm{d} \Theta \\
& +\int_{\Theta_{m}}^{\Theta_{2}^{\prime}} \frac{\partial_{\Theta} B_{0}}{B_{0}^{2}} \frac{2 \varepsilon-\mu B_{0}}{\sqrt{2\left(\varepsilon-\mu B_{0}-\mu \alpha B_{1, \max }\right)}} \mathrm{d} \Theta
\end{aligned}
$$


where $\partial_{\Theta} B_{0}\left(\Theta_{m}\right)=0$. Therefore, $\partial_{\Theta} B_{0}(\Theta)<0$ for $\Theta \epsilon$ $\left[\Theta_{1}^{\prime}, \Theta_{m}\right)$ and $\partial_{\Theta} B_{0}(\Theta)>0$ for $\Theta \in\left(\Theta_{m}, \Theta_{2}^{\prime}\right]$. The minimum of $B_{1}(\Theta)$ in $\left[\Theta_{1}^{\prime}, \Theta_{m}\right.$ ) has been denoted by $B_{1 \text {,min }}$ and the maximum of $B_{1}(\Theta)$ in $\left(\Theta_{m}, \Theta_{2}^{\prime}\right]$ by $B_{1, \max }$. Note that by performing these integrals in $\left[\Theta_{1}^{\prime}, \Theta_{2}^{\prime}\right]$ we have been able to give bounds for the integrand, that tends to infinity at $\Theta=\Theta_{1}$ and $\Theta=\Theta_{2}$.

Let us manipulate the first term on the right side of (63). Trivially,

$$
\begin{aligned}
\int_{\Theta_{1}^{\prime}}^{\Theta_{m}} & \frac{\partial_{\Theta} B_{0}}{B_{0}^{2}} \frac{2 \varepsilon-\mu B_{0}}{\sqrt{2\left(\varepsilon-\mu B_{0}-\mu \alpha B_{1, \min }\right)}} \mathrm{d} \Theta \\
= & \int_{\Theta_{1}^{\prime}}^{\Theta_{m}} \frac{\partial_{\Theta} B_{0}}{B_{0}^{2}} \frac{2 \varepsilon-2 \mu \alpha B_{1, \min }-\mu B_{0}}{\sqrt{2\left(\varepsilon-\mu B_{0}-\mu \alpha B_{1, \min }\right)}} \mathrm{d} \Theta \\
& +\int_{\Theta_{1}^{\prime}}^{\Theta_{m}} \frac{\partial_{\Theta} B_{0}}{B_{0}^{2}} \frac{2 \mu \alpha B_{1, \min }}{\sqrt{2\left(\varepsilon-\mu B_{0}-\mu \alpha B_{1, \min }\right)}} \mathrm{d} \Theta .
\end{aligned}
$$

The integrand of the first term on the right side is an exact differential, whereas the second term is expressed in a useful way after an integration by parts. The result is

$$
\begin{aligned}
& \int_{\Theta_{1}^{\prime}}^{\Theta_{m}} \frac{\partial_{\Theta} B_{0}}{B_{0}^{2}} \frac{2 \varepsilon-\mu B_{0}}{\sqrt{2\left(\varepsilon-\mu B_{0}-\mu \alpha B_{1, \min }\right)}} \mathrm{d} \Theta \\
& =-\frac{1}{B_{0}\left(\Theta_{m}\right)} \sqrt{2\left(\varepsilon-\mu B_{0}\left(\Theta_{m}\right)-\mu \alpha B_{1, \min }\right)} \\
& \quad+\frac{1}{B_{0}\left(\Theta_{1}^{\prime}\right)} \sqrt{2\left(\varepsilon-\mu B_{0}\left(\Theta_{1}^{\prime}\right)-\mu \alpha B_{1, \min }\right)} \\
& \quad-\frac{2 \alpha B_{1, \min }}{B_{0}^{2}\left(\Theta_{m}\right)} \sqrt{2\left(\varepsilon-\mu B_{0}\left(\Theta_{m}\right)-\mu \alpha B_{1, \min }\right)} \\
& +\frac{2 \alpha B_{1, \min }}{B_{0}^{2}\left(\Theta_{1}^{\prime}\right)} \sqrt{2\left(\varepsilon-\mu B_{0}\left(\Theta_{1}^{\prime}\right)-\mu \alpha B_{1, \min }\right)} \\
& +\int_{\Theta_{1}^{\prime}}^{\Theta_{m}} \sqrt{2\left(\varepsilon-\mu B_{0}(\Theta)-\mu \alpha B_{1, \min }\right)} \partial_{\Theta}\left(\frac{\alpha B_{1, \min }}{B_{0}^{2}}\right) \mathrm{d} \Theta .
\end{aligned}
$$

The three last terms (and hence the second term on the right side of (64)) are clearly $O(\alpha)$. Analogous manipulations on the last term of (63) give

$$
\begin{aligned}
\int_{\Theta_{1}^{\prime}}^{\Theta_{2}^{\prime}} & \frac{\partial_{\Theta} B_{0}}{B_{0}^{2}} \frac{2 \varepsilon-\mu B_{0}}{\sqrt{2\left(\varepsilon-\mu B_{0}-\mu \alpha B_{1}\right)}} \mathrm{d} \Theta \\
\leqslant & \frac{1}{B_{0}\left(\Theta_{m}\right)} \sqrt{2\left(\varepsilon-\mu B_{0}\left(\Theta_{m}\right)-\mu \alpha B_{1, \max }\right)} \\
& -\frac{1}{B_{0}\left(\Theta_{m}\right)} \sqrt{2\left(\varepsilon-\mu B_{0}\left(\Theta_{m}\right)-\mu \alpha B_{1, \min }\right)} \\
& +\frac{1}{B_{0}\left(\Theta_{1}^{\prime}\right)} \sqrt{2\left(\varepsilon-\mu B_{0}\left(\Theta_{1}^{\prime}\right)-\mu \alpha B_{1, \min }\right)} \\
& -\frac{1}{B_{0}\left(\Theta_{2}^{\prime}\right)} \sqrt{2\left(\varepsilon-\mu B_{0}\left(\Theta_{2}^{\prime}\right)-\mu \alpha B_{1, \max }\right)} \\
= & O\left(\sqrt{\frac{|\alpha|}{K}}\right) .
\end{aligned}
$$

To write the last equality we have employed that the combination of the first two terms on the right side of (66) is $O(\alpha)$. As for the last two terms, we have used that $\varepsilon-\mu B_{0}(\Theta)>|\alpha| K$ when $\Theta \in\left[\Theta_{1}^{\prime}, \Theta_{2}^{\prime}\right]$ and that $B_{0}\left(\Theta_{2}^{\prime}\right)-$ $B_{0}\left(\Theta_{1}^{\prime}\right)=O(\alpha)$. The latter is an immediate consequence of $\tilde{B}\left(\Theta_{1}^{\prime}\right)=\tilde{B}\left(\Theta_{2}^{\prime}\right)$. Thus,

$$
\begin{aligned}
& \frac{1}{B_{0}\left(\Theta_{1}^{\prime}\right)} \sqrt{2\left(\varepsilon-\mu B_{0}\left(\Theta_{1}^{\prime}\right)-\mu \alpha B_{1, \min }\right)} \\
& -\frac{1}{B_{0}\left(\Theta_{2}^{\prime}\right)} \sqrt{2\left(\varepsilon-\mu B_{0}\left(\Theta_{2}^{\prime}\right)-\mu \alpha B_{1, \max }\right)}=O\left(\sqrt{\frac{|\alpha|}{K}}\right) .
\end{aligned}
$$

Hence, we have shown that

$$
\begin{aligned}
& 2 \int_{\Theta_{1}}^{\Theta_{2}} v_{\psi, \sigma}^{(0)}\left[\left|v_{\|}\right|(\hat{\boldsymbol{b}} \cdot \nabla \Theta)^{(0)}\right]^{-1} \mathrm{~d} \Theta \\
& =-2 \frac{m_{\sigma} c J}{Z_{\sigma} e \Psi_{p}^{\prime}} \int_{\Theta_{1}}^{\Theta_{1}^{\prime}}\left(\frac{\partial_{\Theta} B_{0}}{B_{0}^{2}} \frac{2 \varepsilon-\mu B_{0}}{\sqrt{2\left(\varepsilon-\mu B_{0}-\mu \alpha B_{1}\right)}}\right. \\
& \left.\quad-\frac{\partial_{\Theta} \tilde{B}}{\tilde{B}^{2}} \frac{2 \varepsilon-\mu \tilde{B}}{\sqrt{2(\varepsilon-\mu \tilde{B})}}\right) \mathrm{d} \Theta \\
& -2 \frac{m_{\sigma} c J}{Z_{\sigma} e \Psi_{p}^{\prime}} \int_{\Theta_{2}^{\prime}}^{\Theta_{2}}\left(\frac{\partial_{\Theta} B_{0}}{B_{0}^{2}} \frac{2 \varepsilon-\mu B_{0}}{\sqrt{2\left(\varepsilon-\mu B_{0}-\mu \alpha B_{1}\right)}}\right. \\
& \left.-\frac{\partial_{\Theta} \tilde{B}}{2 \varepsilon-\mu \tilde{B}} \frac{\tilde{B}^{2}}{\sqrt{2(\varepsilon-\mu \tilde{B})}}\right) \mathrm{d} \Theta+O\left(\sqrt{\frac{|\alpha|}{K}}\right) .
\end{aligned}
$$

Using that $\Theta_{1}^{\prime}-\Theta_{1}=O(\alpha K)$ and expanding $B_{0}(\Theta)=$ $B_{0}\left(\Theta_{1}\right)+\partial_{\Theta} B_{0}\left(\Theta_{1}\right)\left(\Theta-\Theta_{1}\right)+O\left(\left(\Theta-\Theta_{1}\right)^{2}\right)$, it is easy to demonstrate that

$\int_{\Theta_{1}}^{\Theta_{1}^{\prime}}\left(\frac{\partial_{\Theta} B_{0}}{B_{0}^{2}} \frac{2 \varepsilon-\mu B_{0}}{\sqrt{2\left(\varepsilon-\mu B_{0}-\mu \alpha B_{1}\right)}}\right.$

$$
\left.-\frac{\partial_{\Theta} \tilde{B}}{\tilde{B}^{2}} \frac{2 \varepsilon-\mu \tilde{B}}{\sqrt{2(\varepsilon-\mu \tilde{B})}}\right) \mathrm{d} \Theta
$$

$=\frac{\partial_{\Theta} B_{0}\left(\Theta_{1}\right)}{B_{0}^{2}\left(\Theta_{1}\right)}\left(2 \varepsilon-\mu B_{0}\left(\Theta_{1}\right)\right)$

$\times \int_{0}^{\Theta_{1}^{\prime}-\Theta_{1}}\left(\frac{1}{\sqrt{2\left[-\mu \partial_{\Theta} B_{0}\left(\Theta_{1}\right) \Delta-\mu \alpha\left(B_{1}\left(\Theta_{1}+\Delta\right)-B_{1}\left(\Theta_{1}\right)\right)\right]}}\right.$

$\left.-\frac{1}{\sqrt{-2 \mu \partial_{\Theta} B_{0}\left(\Theta_{1}\right) \Delta}}\right) \mathrm{d} \Delta+O(\alpha K)$

where the change of variable $\Delta:=\Theta-\Theta_{1}$ has been performed. Noting that $B_{1}$ can be extended to $\Theta \in(-\infty, \infty)$ without problem and Taylor expanding in $\alpha / \Delta$, one gets

$$
\begin{aligned}
\int_{\Theta_{1}^{\prime}-\Theta_{1}}^{\infty} & \left(\frac{1}{\sqrt{2\left[-\mu \partial_{\Theta} B_{0}\left(\Theta_{1}\right) \Delta-\mu \alpha\left(B_{1}\left(\Theta_{1}+\Delta\right)-B_{1}\left(\Theta_{1}\right)\right)\right]}}\right) \mathrm{d} \Delta \\
& -\frac{1}{\sqrt{-2 \mu \partial_{\Theta} B_{0}\left(\Theta_{1}\right) \Delta}} \\
& \sim \int_{\Theta_{1}^{\prime}-\Theta_{1}}^{\infty} \frac{\alpha}{\Delta^{3 / 2}} \mathrm{~d} \Delta=O\left(\sqrt{\frac{|\alpha|}{K}}\right)
\end{aligned}
$$


Similar considerations for the integral over $\left[\Theta_{2}^{\prime}, \Theta_{2}\right]$ in (68) yield, finally,

$$
\begin{aligned}
& 2 \int_{\Theta_{1}}^{\Theta_{2}} v_{\psi, \sigma}^{(0)}\left[\left|v_{\|}\right|(\hat{b} \cdot \nabla \Theta)^{(0)}\right]^{-1} \mathrm{~d} \Theta \\
& =-2 \frac{m_{\sigma} c J}{Z_{\sigma} e \Psi_{p}^{\prime}} \frac{\partial_{\Theta} B_{0}\left(\Theta_{1}\right)}{B_{0}^{2}\left(\Theta_{1}\right)}\left(2 \varepsilon-\mu B_{0}\left(\Theta_{1}\right)\right) \\
& \times \int_{0}^{\infty}\left(\frac{1}{\sqrt{2\left[-\mu \partial_{\Theta} B_{0}\left(\Theta_{1}\right) \Delta-\mu \alpha\left(B_{1}\left(\Theta_{1}+\Delta\right)-B_{1}\left(\Theta_{1}\right)\right)\right]}}\right. \\
& \left.-\frac{1}{\sqrt{-2 \mu \partial_{\Theta} B_{0}\left(\Theta_{1}\right) \Delta}}\right) \mathrm{d} \Delta \\
& -2 \frac{m_{\sigma} c J}{Z_{\sigma} e \Psi_{p}^{\prime}} \frac{\partial_{\Theta} B_{0}\left(\Theta_{2}\right)}{B_{0}^{2}\left(\Theta_{2}\right)}\left(2 \varepsilon-\mu B_{0}\left(\Theta_{2}\right)\right) \\
& \times \int_{0}^{\infty}\left(\frac{1}{\sqrt{2\left[\mu \partial_{\Theta} B_{0}\left(\Theta_{2}\right) \Delta-\mu \alpha\left(B_{1}\left(\Theta_{2}-\Delta\right)-B_{1}\left(\Theta_{2}\right)\right)\right]}}\right. \\
& \left.-\frac{1}{\sqrt{2 \mu \partial_{\Theta} B_{0}\left(\Theta_{2}\right) \Delta}}\right) \mathrm{d} \Delta+O\left(\sqrt{\frac{|\alpha|}{K}}\right)+O(\alpha K) .
\end{aligned}
$$

By choosing $K \sim|\alpha|^{-1 / 3}$, the error is minimized and the resulting corrections in (71) are $O\left(|\alpha|^{2 / 3}\right)$. Written this way, it is manifest that if $\partial_{\Theta} B_{1}(\Theta) \sim \alpha^{-1} B_{1}(\Theta) / L_{0}$ as assumed in this section, then the right-hand side of (71), and therefore the numerator of (54), scales with $|\alpha|^{1 / 2}$. For the bounce time we have

$$
\tau_{b}=2 \int_{\Theta_{1}}^{\Theta_{2}}\left[\left|v_{\|}\right| \hat{b} \cdot \nabla \Theta\right]^{-1} \mathrm{~d} \Theta \sim \frac{L_{0}}{v_{t \sigma}},
$$

so that the bounce-averaged radial magnetic drift of particles trapped in large wells exhibits the scaling announced in (53).

We point out that particles trapped in a large well, but passing sufficiently close to a new $X$ point created by the perturbation, present some peculiarities because the bounce time may be arbitrarily large. Since there are very few of them, we discuss the topic in subsection 4.2. Particles barely trapped in secondary wells exhibit the same phenomenon and represent a significant fraction of all particles trapped in such wells.

\subsection{Secondary wells}

Consider a particle trapped in one of the small secondary wells of figure 1. The size of the new wells is $L_{1} \sim|\alpha| L_{0}$ and the characteristic parallel velocity of particles trapped in them is $v_{||} \sim|\alpha|^{1 / 2} v_{t \sigma}$. Then, it is straightforward to realize that

$$
\overline{v_{\psi, \sigma}} \approx v_{\psi, \sigma}^{(0)}\left(\Theta_{1,0}\right) \sim \epsilon_{\sigma} v_{t \sigma}|\nabla \psi|,
$$

where $\Theta_{1,0}$ is one of the bounce points for $\boldsymbol{B}_{0}$, i.e. a solution of $\varepsilon-\mu B_{0}\left(\Theta_{1,0}\right)=0$. To find (73) we have Taylor expanded $v_{\psi, \sigma}^{(0)}(\Theta)$ around $\Theta_{1,0}$.

Next, we comment on a subtle point. Whereas (73) is correct for all particles trapped in secondary wells, these particles can be split into two families as the scaling with $\alpha$ of their bounce time is concerned. For a typical particle trapped in a secondary well,

$$
\oint \frac{v_{\psi, \sigma}^{(0)}}{v_{||} \hat{\boldsymbol{b}} \cdot \nabla \Theta} \mathrm{d} \Theta \sim|\alpha|^{1 / 2} \epsilon_{\sigma} L_{0}|\nabla \psi|
$$

and

$$
\tau_{b}=\oint \frac{1}{v_{\|} \hat{\boldsymbol{b}} \cdot \nabla \Theta} \mathrm{d} \Theta \sim \frac{L_{1}}{|\alpha|^{1 / 2} v_{t \sigma}} \sim \frac{|\alpha|^{1 / 2} L_{0}}{v_{t \sigma}},
$$

which, of course, yield (73). But there is another interesting type of trajectories, whose discussion is more convenient in coordinates $\{\boldsymbol{R}, \varepsilon, \lambda, s\}$, where

$$
\lambda:=\mu \mathcal{B}_{0} / \varepsilon
$$

is the pitch-angle and $\mathcal{B}_{0}:=\sqrt{\left\langle B^{2}\right\rangle_{\psi}}$. In these coordinates, the parallel velocity reads

$$
v_{\|}=s \sqrt{2 \varepsilon} \sqrt{1-\lambda \frac{B}{\mathcal{B}_{0}}} .
$$

The perturbation $\boldsymbol{B}_{1}$ has created new $\mathrm{X}$ points in phase space, one of which is clearly seen in the gray area of figure 2 . Define $\lambda_{M}=\mathcal{B}_{0} / B_{M}$, where $B_{M}=B\left(\Theta_{M}\right)$ and $\Theta_{M}$ is the value of $\Theta$ at which the $X$ point is located. Since it corresponds to a local maximum of $B(\Theta)$, it is not difficult realize that trajectories with $\lambda=\lambda_{M}$ present a logarithmic divergence in

$$
\oint \frac{v_{\psi, \sigma}^{(0)}}{v_{\|} \hat{\boldsymbol{b}} \cdot \nabla \Theta} \mathrm{d} \Theta
$$

and in the integral that gives the bounce time,

$$
\tau_{b}=\oint \frac{1}{v_{\|} \hat{\boldsymbol{b}} \cdot \nabla \Theta} \mathrm{d} \Theta .
$$

Let us be more precise. If we define $\delta \lambda:=\lambda-\lambda_{M}$, then, for a particle trapped in the secondary well, $\delta \lambda$ is positive. And if $\delta \lambda \lesssim|\alpha|$, one has

$$
\oint \frac{v_{\psi, \sigma}^{(0)}}{v_{||} \hat{\boldsymbol{b}} \cdot \nabla \Theta} \mathrm{d} \Theta \sim|\alpha|^{1 / 2} \ln \left|\frac{\alpha}{\delta \lambda}\right| \epsilon_{\sigma} L_{0}|\nabla \psi|
$$

and

$$
\tau_{b} \sim|\alpha|^{1 / 2} \ln \left|\frac{\alpha}{\delta \lambda}\right| \frac{L_{0}}{v_{t \sigma}} .
$$

It is not difficult to derive these scalings by noting that $\partial_{\Theta}^{2} B(\Theta) \sim \alpha^{-1} B_{0}$, that in a neighborhood of $\lambda_{M}$ and $\Theta_{M}$ the expression (77) for the parallel velocity can be approximated by

$$
v_{\|} \approx s \sqrt{\frac{2 \varepsilon B_{M}}{\mathcal{B}_{0}}} \sqrt{\frac{\lambda_{M}\left|\partial_{\Theta}^{2} B\left(\Theta_{M}\right)\right|}{2 B_{M}}\left(\Theta-\Theta_{M}\right)^{2}-\delta \lambda},
$$

and by observing that the integrals involved in the computation of (80) and (81) are dominated by a region of size $O(\alpha)$ in $\Theta$.

Physically, this result means that particles with small $\delta \lambda$ have large bounce times and, for $\delta \lambda$ strictly equal to 0 , the particle never reaches the bounce point at $\Theta=\Theta_{M}$. However, the ratio of (80) and (81) is such that (73) is satisfied. Anyway, the logarithmic corrections do not affect the size of the distribution function given in section 5 , and the same is true for particles almost trapped in the secondary well briefly mentioned at the end of subsection 4.1. 


\section{Scaling of the distribution function and the flux-surface averaged radial electric current}

We have seen that $\overline{v_{\psi, \sigma}}$ scales differently with $\alpha$ depending on whether the particle is trapped in a large well or trapped in a small well. In order to determine the asymptotic behavior with $\alpha$ of $G_{\sigma}^{[-1]}$, we need to solve the problem independently in several regions of phase space, these regions differing mostly on their characteristic values for $v_{\|}$. Then, global properties will impose matching conditions on the different pieces of $G_{\sigma}^{[-1]}$. Specifically, we divide the phase space in four regions that are shown in figure 2 and will be described in more detail below.

From now on, we assume that the plasma consists of electrons and singly-charged ions, and solve for the ions. Then, the equation to be solved for trapped trajectories is

$$
\Upsilon_{\sigma} \overline{v_{\psi, \mathrm{i}}} F_{\mathrm{i} 0}=\overline{C_{\mathrm{ii}}\left[G_{\mathrm{i}}^{[-1]}\right]},
$$

where we have employed that the ion-electron collision term is small by $\sqrt{m_{\mathrm{e}} / m_{\mathrm{i}}} \ll 1$. A remark about our assumptions on the value of the collisionality is in order here. Equation (83) is correct for trapped particles whose bounce frequency $\tau_{b}^{-1}$ is much larger than their effective collision frequency $v_{\text {eff }}$. A particle trapped in a large well of size $L_{0}$ has $v_{\|} \sim v_{\mathrm{ti}}$, $\tau_{b}^{-1} \sim v_{\text {ti }} / L_{0}$ and $v_{\text {eff }} \sim v_{\mathrm{i}}$. Hence, for them, $v_{\text {eff }} \ll \tau_{b}^{-1}$ is equivalent to

$$
\frac{v_{\mathrm{i}} L_{0}}{v_{\mathrm{ti}}} \ll 1,
$$

which is what one usually understands by low collisionality regime. However, a particle trapped in a secondary well of size $L_{1} \sim|\alpha| L_{0}$ has a parallel velocity $v_{\|} \sim|\alpha|^{1 / 2} v_{\mathrm{ti}}$, a bounce frequency $\tau_{b}^{-1} \sim|\alpha|^{-1 / 2} v_{\mathrm{ti}} / L_{0}$ and an effective collision frequency $v_{\text {eff }} \sim v_{\mathrm{i}} / \alpha$. Then, the condition of small collisionality for these particles amounts to requiring

$$
\frac{v_{\mathrm{i}} L_{0}}{\alpha^{1 / 2} v_{\mathrm{ti}}} \ll 1
$$

which is more demanding that (84). We assume that both (84) and (85) are satisfied so that (83) is the equation determining the distribution function for all trapped trajectories, i.e. all trapped particles are in a $1 / v$ regime (except for the collisional layer shown in figure 2).

Let us start by determining the distribution function in the region corresponding to a small, secondary well, denoted by region I. Its size is of order $L_{1} \sim|\alpha| L_{0}$ and the associated parallel velocities are $v_{\|} \sim|\alpha|^{1 / 2} v_{t \sigma}$. The pitch-angle scattering term dominates in the collision operator of the right-hand side of (83) because $v_{\|}$and $\mu$ have very different scales, leaving us with

$$
\Upsilon_{\sigma} \overline{v_{\psi, i}} F_{\mathrm{i} 0}=\overline{\frac{v_{\|}}{2 \varepsilon \tau_{\perp}} \frac{\mathcal{B}_{0}}{B} \partial_{\lambda}\left(v_{\|} \lambda \partial_{\lambda} G_{\mathrm{i}}^{I}\right)} .
$$

Here, $G_{\mathrm{i}}^{\mathrm{I}}$ stands for the lowest order piece in $\alpha$ of $G_{\mathrm{i}}^{[-1]}$ in region I. The explicit expression for the perpendicular collisional time is

$$
\frac{1}{\tau_{\perp}(\varepsilon)}=\frac{8 \pi e^{4} n_{\mathrm{i}} \ln \Lambda}{m_{\mathrm{i}}^{2}(2 \varepsilon)^{3 / 2}}\left[\Phi\left(\sqrt{m_{\mathrm{i}} \varepsilon / T_{\mathrm{i}}}\right)-\beta\left(\sqrt{m_{\mathrm{i}} \varepsilon / T_{\mathrm{i}}}\right)\right],
$$

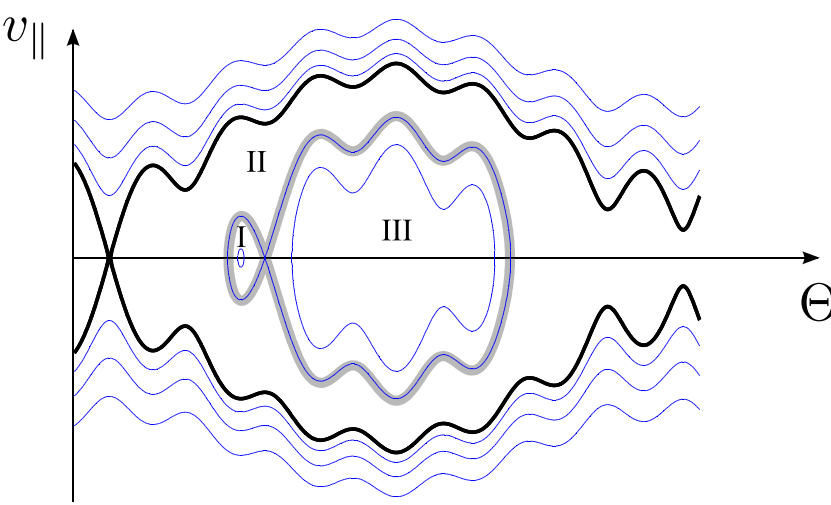

Figure 2. Contours of constant kinetic energy and magnetic moment for the perturbed quasisymmetric field of figure 1 . The regions into which phase space is divided for the calculation of section 5 are shown. Regions I, II and III are labeled. Region IV is the gray area surrounding regions I and III.

where $\ln \Lambda$ is the Coulomb logarithm,

$$
\Phi(x)=\frac{2}{\sqrt{\pi}} \int_{0}^{x} e^{-y^{2}} \mathrm{~d} y
$$

and

$$
\beta(x)=\frac{\Phi(x)-x \Phi^{\prime}(x)}{2 x^{2}} .
$$

From (86) one immediately obtains

$$
\begin{aligned}
\int_{\Theta_{1}}^{\Theta_{2}} & \frac{\Upsilon_{\sigma} v_{\psi, \mathrm{i}}}{\left|v_{||}\right| \hat{\boldsymbol{b}} \cdot \nabla \Theta} F_{\mathrm{i} 0} \mathrm{~d} \Theta \\
= & \int_{\Theta_{1}}^{\Theta_{2}} \frac{\mathcal{B}_{0}}{2 \varepsilon \tau_{\perp} \boldsymbol{B} \cdot \nabla \Theta} \partial_{\lambda}\left(\left|v_{\|}\right| \lambda \partial_{\lambda} G_{\mathrm{i}}^{\mathrm{I}}\right) \mathrm{d} \Theta,
\end{aligned}
$$

$\Theta_{1}$ and $\Theta_{2}$ being the bounce points. Hence,

$$
\begin{aligned}
\partial_{\lambda} G_{\mathrm{i}}^{\mathrm{I}} & =\left(\int_{\Theta_{1}}^{\Theta_{2}} \frac{\mathcal{B}_{0}}{2 \varepsilon \tau_{\perp} \boldsymbol{B} \cdot \nabla \Theta}\left|v_{\|}\right| \mathrm{d} \Theta\right)^{-1} \\
& \times \frac{1}{\lambda} \int_{\lambda_{b}}^{\lambda} \int_{\Theta_{1}}^{\Theta_{2}} \frac{\Upsilon_{\sigma} v_{\psi, \mathrm{i}}}{\left|v_{\|}\right| \hat{\boldsymbol{b}} \cdot \nabla \Theta} F_{\mathrm{i} 0} \mathrm{~d} \Theta
\end{aligned}
$$

where we have used that $G_{\mathrm{i}}^{\mathrm{I}}$ is constant over the orbit and we have assumed the regularity condition $\left.v_{\|} \partial_{\lambda} G_{\mathrm{i}}^{\mathrm{I}}\right|_{\lambda_{b}}=0$ at the bottom of the well, $\lambda_{b}=\mathcal{B}_{0} / B_{b}$, with $B_{b}$ the minimum value of $B$ in the well. Observe that (91) completely determines $\partial_{\lambda} G_{\mathrm{i}}^{\mathrm{I}}$ inside the secondary well. Finally, noting that the size of the well is $O(\alpha)$ in the pitch-angle coordinate, one learns that

$$
\partial_{\lambda} G_{\mathrm{i}}^{\mathrm{I}} \sim \nu_{* \mathrm{i}}^{-1} \epsilon_{\mathrm{i}} F_{\mathrm{i} 0} .
$$

It is easy to convince oneself that the logarithmic corrections in (80) associated to particles barely trapped in the secondary well give subdominant contributions after performing the integral over $\lambda$ in equation (91).

We have obtained $\partial_{\lambda} G_{i}^{\mathrm{I}}$, but we do not know the size of $G_{\mathrm{i}}^{\mathrm{I}}$ yet. For this, we need to know the distribution function in regions II and III and integrate in $\lambda$ from regions II and III towards $\lambda_{b}$.

In regions II and III one cannot simplify the collision operator and the solution cannot be given as explicitly as in 
region I, but it can be found numerically. Here, we only need to use that, due to the results of section 4, equation (83) gives

$$
\begin{aligned}
& G_{\mathrm{i}}^{\mathrm{II}} \sim|\alpha|^{1 / 2} v_{* \mathrm{i}}^{-1} \epsilon_{\mathrm{i}} F_{\mathrm{i} 0}, \\
& G_{\mathrm{i}}^{\mathrm{III}} \sim|\alpha|^{1 / 2} v_{* \mathrm{i}}^{-1} \epsilon_{\mathrm{i}} F_{\mathrm{i} 0}
\end{aligned}
$$

and also

$$
\begin{aligned}
& \partial_{\lambda} G_{\mathrm{i}}^{\mathrm{II}} \sim|\alpha|^{1 / 2} \nu_{* \mathrm{i}}^{-1} \epsilon_{\mathrm{i}} F_{\mathrm{i} 0}, \\
& \partial_{\lambda} G_{\mathrm{i}}^{\mathrm{III}} \sim|\alpha|^{1 / 2} \nu_{* \mathrm{i}}^{-1} \epsilon_{\mathrm{i}} F_{\mathrm{i} 0} .
\end{aligned}
$$

Now, recall that at the end of section 3 we proved that $G_{i}^{[-1]}=0$ for passing particles to lowest order in $\alpha$. Therefore, the boundary condition needed to solve for $G_{\mathrm{i}}^{\mathrm{II}}$ is $G_{\mathrm{i}}^{\mathrm{II}}=0$ at the passing/trapped interface. Finally, $G_{\mathrm{i}}^{\mathrm{III}}$ is determined by imposing continuity between regions II and III (we cannot discard the existence of a discontinuity in $\partial_{\lambda} G_{\mathrm{i}}$ between regions II and III).

The relation between the different regions is provided by the emergence of a collisional layer in region IV, the thin gray area in figure 2 . In this region the bounce-averaged equation (83) is not suitable because particles collide too frequently. In the layer the parallel streaming and collision terms in the drift-kinetic equation (9) balance each other, giving

$$
v_{\|} \hat{\boldsymbol{b}} \cdot \nabla \sim v_{\mathrm{i}} \partial_{\xi}^{2} .
$$

Equivalently,

$$
v_{\|} \hat{\boldsymbol{b}} \cdot \nabla \sim \frac{v_{\mathrm{i}}}{\delta \xi^{2}},
$$

where $\xi=v_{\|} / v_{\mathrm{ti}}$ and $\delta \xi$ stands for the width of the layer. The distribution function has large parallel velocity derivatives in the collisional layer, and the pitch-angle scattering piece of the collision operator dominates. The secondary well has a typical size $L_{1} \sim|\alpha| L_{0}$ and particles trapped in it have typical parallel velocities $v_{\|} \sim|\alpha|^{1 / 2} v_{\mathrm{ti}}$. Then, (98) yields

$\delta \xi \sim\left(\frac{v_{\mathrm{i}} L_{1}}{|\alpha|^{1 / 2} v_{\mathrm{ti}}}\right)^{1 / 2} \sim\left(\frac{v_{\mathrm{i}} L_{1}}{|\alpha|^{3 / 2} v_{\mathrm{ti}}}\right)^{1 / 2}|\alpha|^{1 / 2} \ll|\alpha|^{1 / 2}$,

where we have used (85). Hence, the width in $v_{\|}$of the collisional layer around the left lobe of region IV (see figure 2) is much smaller than the typical value of $v_{\|}$at the boundary, as it should. As for the right lobe of region IV, with size $L_{0}$ and typical parallel velocities $v_{\|} \sim v_{\mathrm{ti}}$, one also gets that the width of the layer is consistent,

$$
\delta \xi \sim\left(\frac{v_{\mathrm{i}} L_{0}}{v_{\mathrm{ti}}}\right)^{1 / 2} \ll 1,
$$

due to (84).

The equation in the collisional boundary layer is

$$
v_{\|} \hat{\boldsymbol{b}} \cdot \nabla G_{\mathrm{i}}^{\mathrm{IV}}=\frac{v_{\|}}{2 \varepsilon \tau_{\perp}} \frac{\mathcal{B}_{0}}{B} \partial_{\lambda}\left(v_{\|} \lambda \partial_{\lambda} G_{\mathrm{i}}^{\mathrm{IV}}\right) .
$$

Multiplying by $v_{\|}^{-1}$ and integrating along the field line,

$$
\oint \frac{\mathcal{B}_{0}}{2 \varepsilon \tau_{\perp} \boldsymbol{B} \cdot \nabla \Theta} \partial_{\lambda}\left(v_{\|} \lambda \partial_{\lambda} G_{\mathrm{i}}^{\mathrm{IV}}\right) \mathrm{d} \Theta=0,
$$

where $\oint$ stands for the integral over the corresponding trapped orbit. Hence, integrating in $\lambda$ over the collisional layer, we get

$$
\begin{aligned}
\oint & \frac{\mathcal{B}_{0}}{2 \varepsilon \tau_{\perp} \boldsymbol{B} \cdot \nabla \Theta}\left(v_{\|} \lambda \partial_{\lambda} G_{\mathrm{i}}^{I}\right) \mathrm{d} \Theta \\
& +\oint \frac{\mathcal{B}_{0}}{2 \varepsilon \tau_{\perp} \boldsymbol{B} \cdot \nabla \Theta}\left(v_{\|} \lambda \partial_{\lambda} G_{\mathrm{i}}^{\mathrm{II}}\right) \mathrm{d} \Theta \\
& +\oint \frac{\mathcal{B}_{0}}{2 \varepsilon \tau_{\perp} \boldsymbol{B} \cdot \nabla \Theta}\left(v_{\|} \lambda \partial_{\lambda} G_{\mathrm{i}}^{\mathrm{III}}\right) \mathrm{d} \Theta=0
\end{aligned}
$$

where all terms are evaluated at the boundary of region IV.

We already know that $\partial_{\lambda} G_{\mathrm{i}}^{\mathrm{I}} \sim v_{* \mathrm{i}}^{-1} \epsilon_{\mathrm{i}} F_{\mathrm{i} 0}$. Taking into account that in region I $v_{\|} \sim|\alpha|^{1 / 2} v_{\mathrm{ti}}$, and the size of the secondary well is $L_{1} \sim|\alpha| L_{0}$, we deduce that the first term in (103) is $O\left(|\alpha|^{3 / 2}\right)$. Therefore,

$$
\begin{aligned}
& \oint \frac{\mathcal{B}_{0}}{2 \varepsilon \tau_{\perp} \boldsymbol{B} \cdot \nabla \Theta}\left(v_{\|} \lambda \partial_{\lambda} G_{\mathrm{i}}^{\mathrm{II}}\right) \mathrm{d} \Theta \\
& \quad+\oint \frac{\mathcal{B}_{0}}{2 \varepsilon \tau_{\perp} \boldsymbol{B} \cdot \nabla \Theta}\left(v_{\|} \lambda \partial_{\lambda} G_{\mathrm{i}}^{\mathrm{III}}\right) \mathrm{d} \Theta=O\left(|\alpha|^{3 / 2}\right)
\end{aligned}
$$

Since the typical value of the parallel velocity in the terms on the left side of the previous equation is $v_{\|} \sim v_{\mathrm{ti}}$, we infer that the jump of $\partial_{\lambda} G_{\mathrm{i}}$ between regions II and III is $O\left(|\alpha|^{3 / 2} v_{* \mathrm{i}}^{-1} \epsilon_{\mathrm{i}} F_{\mathrm{i} 0}\right)$. It may seem that this jump is negligible compared to $\partial_{\lambda} G_{\mathrm{i}} \sim|\alpha|^{1 / 2} \nu_{* \mathrm{i}}^{-1} \epsilon_{\mathrm{i}} F_{\mathrm{i} 0}$, but in general there is a number of small wells of order $|\alpha|^{-1}$ in a field line and the accumulation of these discontinuities modifies $G_{\mathrm{i}}$ by a quantity of order $|\alpha|^{1 / 2} v_{* \mathrm{i}}^{-1} \epsilon_{\mathrm{i}} F_{\mathrm{i} 0}$.

We denote the union of regions II and III by region II'. The smallness of the size of the boundary layer (region IV) implies that $G_{\mathrm{i}}^{\mathrm{I}}$ and $G_{\mathrm{i}}^{\mathrm{II}}$ are continuous to lowest order (their derivatives are not). Since we know $\partial_{\lambda} G_{\mathrm{i}}^{\mathrm{I}}$, we can integrate from the boundary of the secondary well towards the bottom $\lambda_{b}$. It is easy to realize that the change in $G_{\mathrm{i}}^{\mathrm{I}}$ is negligible compared to $G_{\mathrm{i}}^{\mathrm{II}}$, giving $G_{\mathrm{i}}^{\mathrm{I}} \sim G_{\mathrm{i}}^{\mathrm{II}} \sim|\alpha|^{1 / 2} \nu_{* \mathrm{i}}^{-1} \epsilon_{\mathrm{i}} F_{\mathrm{i} 0}$. All these scalings work analogously for electrons.

Using the above results and noting that in velocity space the fraction of particles trapped in secondary wells scales as $|\alpha|^{1 / 2}$, we find that all trapped trajectories contribute with the same scaling to the flux-surface averaged radial electric current,

$$
\begin{gathered}
\langle\boldsymbol{J} \cdot \nabla \psi\rangle_{\psi}=\left\langle\int G_{\mathrm{i}}^{[-1]} \overline{v_{\psi, \sigma}^{(0)}} \mathrm{d}^{3} v\right\rangle_{\psi}+\ldots \\
=\left\langle\int G_{\mathrm{i}}^{\mathrm{l}} \overline{v_{\psi, \sigma}^{(0)}} \mathrm{d}^{3} v\right\rangle_{\psi}+\left\langle\int G_{\mathrm{i}}^{\mathrm{II}} \overline{v_{\psi, \sigma}^{(0)}} \mathrm{d}^{3} v\right\rangle_{\psi} \\
+\ldots \sim \frac{|\alpha| \epsilon_{\mathrm{i}}^{2} v_{\mathrm{ti}}}{v_{\mathrm{i}} L_{0}} n_{\mathrm{i}} v_{\mathrm{ti}}|\nabla \psi|,
\end{gathered}
$$

where the dots stand for higher-order terms. Here, we have also employed that the size of the well in $\chi$ is of order $|\alpha|$, that the number of small wells on a given magnetic field line is of 
order $|\alpha|^{-1}$, and that the number of lines with small wells is also of order $|\alpha|^{-1}$.

The main result obtained in this paper, equation (105), contradicts the $|\alpha|^{3 / 2}$ scaling typically associated to a symmetric magnetic field where a ripple magnetic field that creates secondary wells has been added [14]. The argument leading to such a scaling is easy and plausible, but the above rigorous treatment shows that it is incorrect. The former is based on the assumption that the secondary wells dominate transport, whereas we have proven that all trapped trajectories contribute the same. Going through similar steps as above, it is easy to show that the scaling $|\alpha|^{3 / 2}$ can be obtained by adopting the assumption that particles trapped in the large wells of $\boldsymbol{B}_{0}$ drift outwards at an unrealistically low rate $O\left(\alpha \epsilon_{\mathrm{i}} v_{\mathrm{ti}}\right)$.

Finally, one might wonder how the rotation criterion (5) is modified if large helicity perturbations are present. When $\alpha \hat{\boldsymbol{b}}_{0} \cdot \nabla B_{1} \sim \hat{\boldsymbol{b}}_{0} \cdot \nabla B_{0}$, we have only treated the $1 / v$ regime, and therefore the comparison has to be carried out with (5) particularized for low collisionality. If one uses (3), condition (5) can be more precisely formulated, giving the rotation criterion

$$
|\alpha|<\sqrt{\nu_{* \mathrm{i}} \epsilon_{\mathrm{i}}}
$$

for small helicity perturbations. The same arguments that lead to this criterion, exposed in [9], can be repeated employing now the scaling (105), yielding the rotation criterion

$$
|\alpha|<v_{* \mathrm{i}} \epsilon_{\mathrm{i}}
$$

for large helicity perturbations. Then, for a fixed value of $\alpha$, flow damping is stronger if large helicity perturbations are not avoided. This should be taken into account in future quasisymmetric stellarator designs.

\section{Conclusions}

Quasisymmetry is an interesting design concept in stellarator research but it is known [7] that it cannot be achieved exactly, even if configurations reasonably close to quasisymmetric are feasible [8]. In the light of these facts, it is important to understand quantitatively how physical features associated to quasisymmetry, such as the possibility to have large equilibrium flows, are affected by small deviations from it. To answer this question, we have calculated how the flux-surface averaged radial electric current (that identically vanishes for a quasisymmetric configuration) deviates from zero depending on the size and other properties of the non-quasisymmetric magnetic field perturbation.

The systematic treatment of the problem started in [9], where the scaling with the size of the perturbation was derived for the least deleterious type of perturbation, one with small spatial gradients. Let the magnetic field be $\boldsymbol{B}=\boldsymbol{B}_{0}+\alpha \boldsymbol{B}_{1}$, where $\boldsymbol{B}_{0}$ is quasisymmetric and $\alpha \boldsymbol{B}_{1}$ a perturbation. If the helicity of the latter is sufficiently small (see the Introduction for a precise statement of the conditions), then

$$
\langle\boldsymbol{J} \cdot \nabla \psi\rangle_{\psi} \sim \alpha^{2} k,
$$

where the form of the factor $k$ depends on the collisionality regime.
More dangerous types of perturbations (again, we refer the reader to the Introduction for the technical details), that should be avoided, if possible, when designing quasisymmetric stellarators, have been the subject of this paper. In general, when the gradient of the perturbation is large one has

$$
\langle\boldsymbol{J} \cdot \nabla \psi\rangle_{\psi} \sim O\left(\alpha^{0}\right),
$$

which amounts to say that the quasisymmetric properties of $\boldsymbol{B}_{0}$ have been lost. However, an intermediate situation between (108) and (109) exists when the gradient of the perturbation is large but it is aligned with the magnetic field lines. Then, one gets

$$
\langle\boldsymbol{J} \cdot \nabla \psi\rangle_{\psi} \sim \frac{|\alpha| \epsilon_{\mathrm{i}}^{2} v_{\mathrm{ti}}}{v_{\mathrm{i}} L_{0}} e n_{\mathrm{i}} v_{\mathrm{ti}}|\nabla \psi|
$$

in the $1 / v$ regime. Such large parallel derivatives are typically associated to the appearance of small secondary wells and it has often been believed in the literature that these small wells dominate transport and that they produce a scaling $|\alpha|^{3 / 2}$. We have shown that the $|\alpha|^{3 / 2}$ scaling is incorrect.

We have also explained why (110) implies that the capability of the stellarator to rotate is reduced with respect to the case in which only small helicity perturbations exist.

\section{Acknowledgments}

This work was supported by EURATOM and carried out within the framework of the EUROfusion Consortium. This project has received funding from the EU Horizon 2020 research and innovation programme. The views and opinions expressed herein do not necessarily reflect those of the European Commission. This research was supported in part by grant ENE2012-30832, Ministerio de Economía y Competitividad, Spain.

\section{References}

[1] Boozer A H 1983 Phys. Fluids 26496

[2] Nührenberg J and Zille R 1988 Phys. Lett. A 129113

[3] Helander P et al 2012 Plasma Phys. Control. Fusion 54124009

[4] de Vries P C, Waidmann G, Donné A J H and Schüller F C 1996 Plasma Phys. Control. Fusion 38467

[5] Mantica P et al 2009 Phys. Rev. Lett. 102175002

[6] Dinklage A et al, LHD Exp. Group, TJ-II Team and W7-AS Team 2013 Nucl. Fusion 53063022

[7] Garren D A and Boozer A H 1991 Phys. Fluids B 32822

[8] Anderson F S B, Almagri A F, Anderson D T, Matthews P G, Talmadge J N and Shohet J L 1995 Fusion Technol. 27273

[9] Calvo I, Parra F I, Velasco J L and Alonso J A 2013 Plasma Phys. Control. Fusion 55125014

[10] Helander P and Simakov A N 2008 Phys. Rev. Lett. 101 145003

[11] Boozer A H 1981 Phys. Fluids 241999

[12] Simakov A N and Helander P 2011 Plasma Phys. Control. Fusion 53024005

[13] Sugama H, Watanabe T H, Nunami M and Nishimura S 2011 Phys. Plasmas 18082505

[14] Ho D D M and Kulsrud R M 1987 Phys. Fluids 30442

[15] Beidler C D et al 2011 Nucl. Fusion 51076001

[16] Simakov A N and Helander P 2009 Phys. Plasmas 16042503 